\title{
Téoros
}

Revue de recherche en tourisme

\section{Le tourisme dans le Vieux-Montréal}

Une fonction au coeur de sa renaissance et de sa réhabilitation

\section{Martin Drouin}

Volume 28, numéro 1, 2009

URI : https://id.erudit.org/iderudit/1024840ar

DOI : https://doi.org/10.7202/1024840ar

Aller au sommaire du numéro

Éditeur(s)

Université du Québec à Montréal

ISSN

0712-8657 (imprimé)

1923-2705 (numérique)

Découvrir la revue

\section{Citer cette note}

Drouin, M. (2009). Le tourisme dans le Vieux-Montréal : une fonction au coeur de sa renaissance et de sa réhabilitation. Téoros, 28(1), 93-96.

https://doi.org/10.7202/1024840ar d'utilisation que vous pouvez consulter en ligne.

https://apropos.erudit.org/fr/usagers/politique-dutilisation/ 


\title{
Le tourisme dans le Vieux-Montréal Une fonction au cœur de sa renaissance et de sa réhabilitation
}

\author{
Martin DROUIN, Ph.D. \\ Coordonnateur de l'Institut du patrimoine \\ Professeur associé \\ Université du Québec à Montréal \\ drouin.martin@uqam.ca
}

En octobre 2008, la Table de concertation du VieuxMontréal, une structure consultative créée en 1993 au lendemain du Forum sur le tourisme dans le Vieux-Montréal coorganisé par la Société immobilière du patrimoine architectural de Montréal (SIMPA) et la Chaire de tourisme de l'École des sciences de la gestion de l'Université du Québec à Montréal, publiait un document intitulé : Le Vieux-Montréal 2017, L'art de vivre montréalais dans une collection historique inscrite dans la modernité. L'objectif de la réflexion menée à l'intérieur de groupes de discussions était de penser le futur du quartier dans un horizon d'une dizaine d'années, choix symbolique correspondant au $375^{\mathrm{e}}$ anniversaire de la fondation de la ville. Trois grandes orientations ont émergé de ce travail : bien vivre, prendre soin de l'héritage historique et oser l'avenir. Le patrimoine architectural, valorisé comme témoignage structuré autour de six époques distinctes de construction, y tient naturellement une place centrale. Toutefois, la volonté d'y intégrer une touche de modernité révèle le désir de renverser la tentation d'imposer une vision passéiste de l'arrondissement. Avec une force encore plus vive, le versant touristique du quartier trouve sa contrepartie dans l'effort de prendre en compte les travailleurs qui s'y rendent chaque jour et, surtout, les résidents qui en ont fait leur cadre de vie. Plus que tout, il s'agit d' "assurer l'équilibre et l'harmonie entre les fonctions et les multiples activités qui se déploient dans le Vieux-Montréal» (Ramacieri, 2008 : 2). À cet égard, la place de tourisme est totalement différente de celle qui avait été pensée au moment où le Vieux-Montréal fut protégé au début des années 1960. Que s’est-il passé entre ces deux moments?

Plusieurs facteurs peuvent expliquer ce changement d'attitude. Tout d'abord, bien de l'eau a coulé sous les ponts depuis cette période épique et la transformation des sensibilités a placé le patrimoine au centre de bien des priorités. Il n'est plus nécessaire de convaincre de la nécessité de sauvegarder l'arrondissement historique, alors que les autorités municipales et provinciales y ont investi des millions de dollars. Depuis plusieurs décennies, la volonté de réimplanter la fonction résidentielle dans le secteur et le désir de réappropriation par les Montréalais ont aussi transformé le rapport à la ville. Une gestion plus attentive des arrondissements historiques a parallèlement pris en compte la fragile harmonie de ces écosystèmes urbains. Enfin, la peur de la muséification et de la folklorisation hante beaucoup d'interventions. Il est cependant déplorable que la fonction touristique soit désormais reléguée à un rôle de second plan, alors qu'il n'y a pas si longtemps elle sauvait pourtant le quartier. Cela n'est pas étonnant. Pour plusieurs, le tourisme est ce pacte avec le diable signé au prix d'une aliénation du patrimoine bien dommageable. Il est cet étrange phénomène valorisé lorsque l'économie chancelle, mais rejeté du revers de la main à mesure que la situation se rétablit. En ce sens, la production d'un document comme celui de la Table de concertation du Vieux-Montréal est une excellente nouvelle, car il pousse à croire que le quartier est en bonne santé, même s'il doit encore faire face à des défis importants. Il n'en demeure pas moins que le tourisme a été fondamental à sa réhabilitation : il a permis la mise en place d'une véritable offre culturelle; il a favorisé la structuration de nouvelles pratiques urbaines; il a enfin favorisé la constitution d'une identité urbaine montréalaise.

Remettons quelques évènements en perspective. Avant l'espoir suscité par la sauvegarde d'un secteur historique dans les années 1960, Montréal avait bien peu à offrir en termes culturel. La ville misait sur d'autres aspects de sa personnalité pour attirer les touristes. L'attractivité montréalaise se définissait par la lorgnette de son dynamisme économique et de son caractère métropolitain. Les visiteurs venaient se laisser happer par le tourbillon d'une grande ville. À cela s'ajoutaient la présence importante d'une population d'origine française et la coloration procurée 


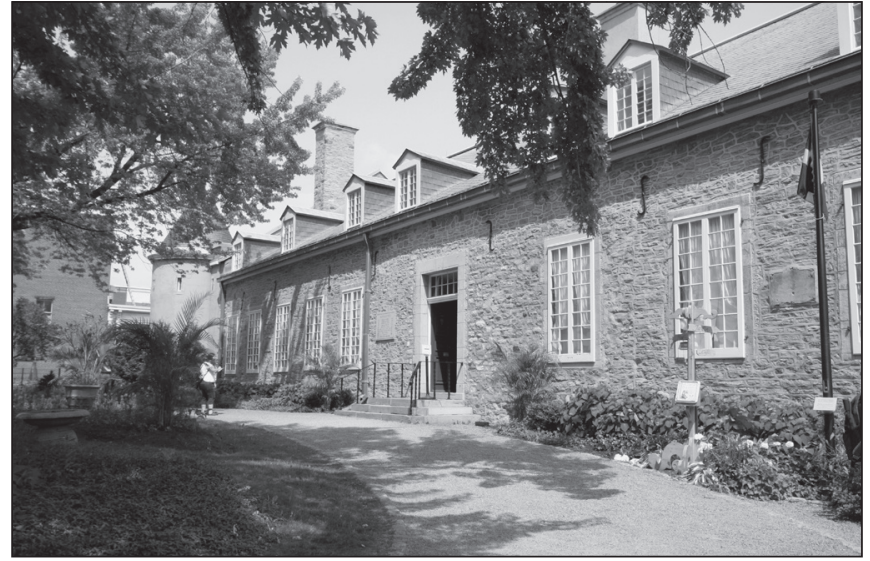

ILLUSTRATION 1 : Le Musée du château de Ramezay

(photo : Martin Drouin).

par une immigration intense. Enfin, la multitude de clochers dans le ciel montréalais donnait à la ville ou un air de piété ou une silhouette particulière capable de piquer la curiosité et d'en étonner plus d'un (Ramsay, 2003). Les festivités de 1917 et de 1942, dates anniversaires de la fondation de la ville, auraient pu, comme ce fut le cas à Québec avec le Tricentenaire de 1908, structurer une véritable offre culturelle. Elles furent plutôt des occasions ratées dans le contexte peu réjouissant des deux guerres mondiales. Il est vrai qu'un premier circuit historique fut organisé dans les rues du Vieux-Montréal (Société historique de Montréal, 1917). Toutefois, offerte une fois par année, le 17 mai, c'est-à-dire la journée anniversaire de l'arrivée de Paul Chomedey de Maisonneuve et de ses compagnons (en 1642), la visite n'avait pas tous les atouts nécessaires pour attirer des curieux! En termes d'équipement culturel, Montréal ne croulait pas davantage sous l'offre. Le Musée des beaux-arts de Montréal (1860), le musée du château de Ramezay (1895), le musée McCord (1921) exposaient leurs collections au côté de quelques autres petites institutions. Au début des années 1960, le constat était sans appel : «Montréal, métropole du Canada, a besoin de musées [et] on le déplore depuis longtemps.» (Prévost, 1963) Alors que le quotidien La Presse publiait une série d'articles sur la question, la situation commençait à évoluer. À cet égard, les commentateurs ont souvent insisté sur l'impact de la venue de l'Exposition universelle de 1967 pour expliquer les changements intervenus à ce moment. Sans en nier l'incidence, il ne faudrait cependant pas oublier la place fondamentale de la sauvegarde et de la réhabilitation du Vieux-Montréal dans le processus de structuration d'une nouvelle offre touristique.

Il est vrai que les deux phénomènes sont liés l'un à l'autre. Au tournant des années 1960, la vitalité économique du secteur appelé aujourd'hui le Vieux-Montréal périclitait. De plus, la valeur de son cadre bâti était encore loin d'être admise par tous. Rappelons seulement que l'inventaire dressé par le Service d'urbanisme ne recensa «que 18 maisons historiques encore debout» dans le secteur

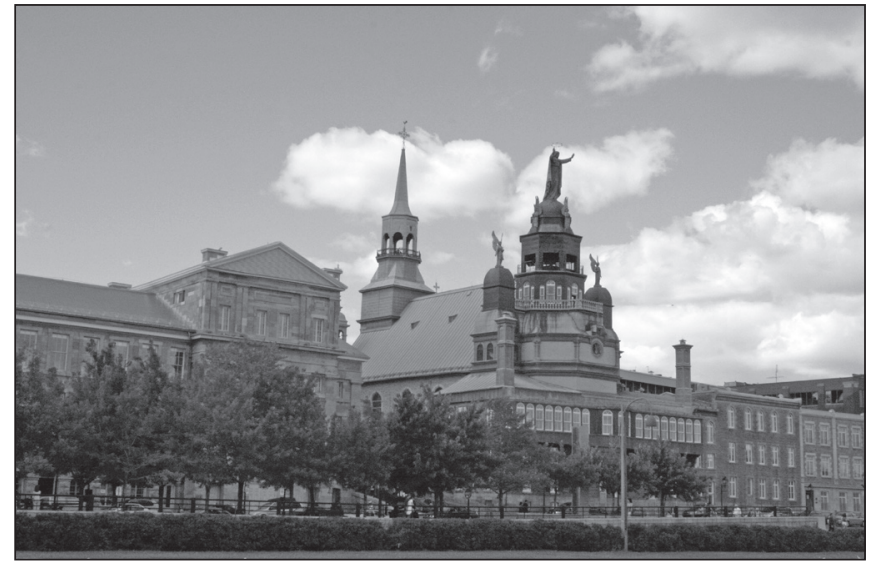

ILLUSTRATION 2 : Chapelle Notre-Dame-du-Bonsecours

(photo : Martin Drouin).

(Mondello, 1961). Une série de menaces vint bousculer les consciences et permit la création de la Commission Jacques-Viger par les autorités municipales en 1962, puis la constitution de l'arrondissement historique par le gouvernement québécois en 1964. Toutefois, l'annonce des célébrations du centenaire de la Confédération canadienne en 1967 et de l'organisation d'une exposition universelle, au moment où Montréal allait célébrer son $325^{\mathrm{e}}$ anniversaire de fondation, s'ajouta aux arguments appuyant le financement d'un projet de restauration (Drouin, 2008). La présence d'un Vieux-Montréal réhabilité dans une foire aux accents futuristes n'était pas sans donner une assise historique à la proposition de représenter la «Terre des hommes». D'ailleurs, fut rapidement abandonnée l'idée d'ériger un village historique canadien sur l'île Sainte-Hélène, non loin de la tour Lévis et de l'ancien fort occupé en partie par le Musée militaire de Montréal (aujourd'hui musée Stewart) qui avait ouvert ses portes en 1955. Les autorités préféraient concentrer leur énergie sur un véritable projet de mise en valeur du Montréal historique, tel qu'il était défini à l'époque. De plus, comme le soulignait le directeur du château de Ramezay, musée situé dans le Vieux-Montréal, "In addition to preserving that which is irreplaceable, the Old Quarter would provide Montreal with greatest tourist attraction.» (Palmer, 1961) Un nouvel usage touristique justifiait l'entreprise et l'importance des fonds qui allaient être investis.

Il n'est donc pas étonnant que l'Office municipal de tourisme, créé en 1961 pour faire valoir les attraits de la métropole en prévision de la tenue de l'Exposition universelle, ait ouvert un bureau d'information sur la rue Notre-Dame. Son directeur, Lucien Bergeron, membre de la Commission Jacques-Viger, s'imposa comme un défenseur convaincu du potentiel touristique du secteur. Pour lui, comme pour d'autres, «les touristes qui visitent Montréal demandent à voir le caractère typiquement français de la ville, le 'Old French Quarter'»(sans auteur, 1963b). C'était ce vieux quartier français que les promoteurs espéraient faire revivre par différentes stratégies. Une politique d'affichage voulut 
d'abord éliminer les enseignes criardes et lumineuses par d'autres plus respectueuses du caractère historique. Une révision de l'éclairage existant passa par la volonté d'installer des lampadaires inspirés de modèles du XIX ${ }^{\mathrm{e}}$ siècle. Un tronçon de la rue Saint-Paul Est retrouva un aspect vieillot avec la pose de pavés. Dans le même secteur, les autorités municipales décidèrent de restaurer l'ancien marché Bonsecours, dont le dôme avait disparu lors d'un incendie. On rénova aussi les places Jacques-Cartier et Vauquelin et sur la première on aménagea un kiosque aux fleurs pour le plus grand plaisir des commentateurs. Au même moment, on organisa les premières visites en calèche. Un membre de la Commission Jacques-Viger suggéra même de cesser l'épandage de sel dans les rues pendant l'hiver, «ce qui rendrait possible [disait-il] la circulation de traîneaux pour les touristes » (Archives de la Ville de Montréal, 1965). L'objectif était d' «historiciser» l'apparence du quartier afin de mieux la faire coller à la conception de l'attrait touristique et à l'idée qu'on se faisait d'un arrondissement historique.

À la pauvreté des installations culturelles soulignées précédemment, la réhabilitation du Vieux-Montréal suscitait, quant à elle, la proposition d'une multitude de projets. L'idée poussée le plus loin fut celle de créer le «village de Maisonneuve», proposée par John David King, conservateur au musée du château de Ramezay et aussi membre de la Commission Jacques-Viger. Concentré dans le secteur est du Vieux-Montréal, le village historique ambitionnait de faire revivre l'atmosphère de la ville coloniale par la conservation ou le déplacement de bâtiments anciens de même que la reconstitution d'une partie du mur de fortification disparu depuis le début du XIX ${ }^{\mathrm{e}}$ siècle (Laliberté, 1961). Dans cette dynamique, les projets de musée d'histoire se succédèrent avec insistance. Un élu déposait un plan pour transformer le vieil édifice de la douane en un musée d'histoire urbaine (sans auteur, 1963a). Le directeur du musée du château de Ramezay souhaitait agrandir ses salles d'exposition dans l'hôtel Rasco, dont les propriétaires cherchaient une nouvelle vocation (Archives de la Ville de Montréal, 11 janvier 1965). La Société historique de Montréal imaginait un immense projet : recycler un magasin-entrepôt de l'Hôtel-Dieu pour exposer les collections d'institutions privées montréalaises et créer, en parallèle, un centre d'archives (Luchaire, 1966). Si ces projets demeurèrent dans les cartons, d'autres virent le jour. La maison Del Vecchio, nouvellement restaurée, accueillit pendant un moment une collection d'armes anciennes puis présenta une sélection de plats en argent ciselé de la maison Birk's. La maison du Calvet, également rénovée, offrit au regard des visiteurs une sélection de meubles canadiens appartenant au Musée des beaux-arts de Montréal. La maison de la Sauvegarde, réhabilitée par la compagnie d'assurance du même nom, ouvrit ses portes à des expositions d'art. La chapelle Notre-Damedu-Bon-Secours proposa un parcours autour de la vie de Marguerite Bourgeoys, tandis que la Banque de Montréal exposa des pièces de monnaie ancienne et d'autres objets liés au monde bancaire. Cette activité muséale, réelle ou imaginée, démontrait bien le rôle symbolique assigné au VieuxMontréal : être le gardien et le médiateur de la mémoire

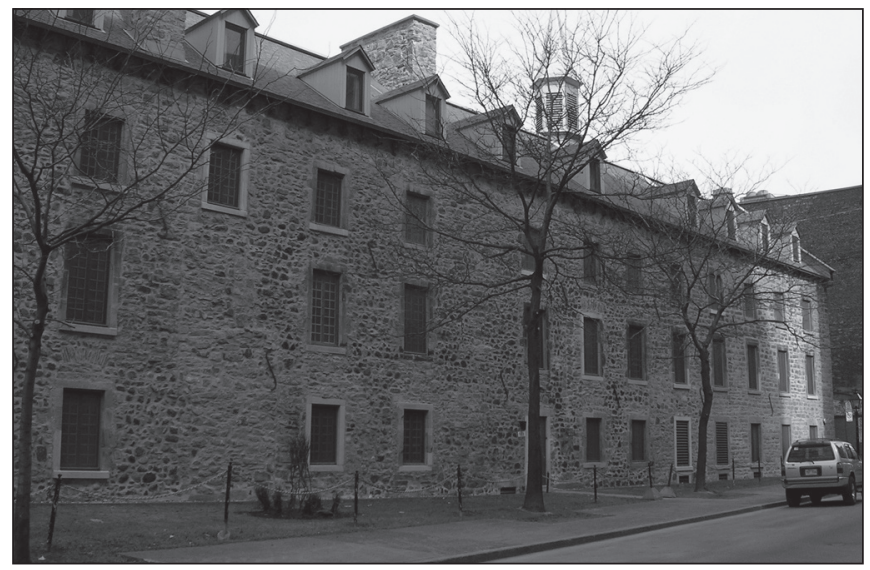

ILLUSTRATION 3 : Ancien Hôpital général des Sœurs grises

(photo : Martin Drouin).

urbaine. Cette fonction, l'éditorialiste de La Presse, Alfred Ayotte (1964), aussi membre de la Commission JacquesViger, l'exprima clairement lorsqu'il souhaita que « Le Vieux Montréal [devienne] avec le temps un musée de plein air où Montréalais et visiteurs pourront s'y promener en respirant l'air du passé, en évoquant les souvenirs d'autrefois.» Un quartier à vocation culturelle se construisait à mesure que les bâtiments étaient restaurés.

La grande nouveauté fut de faire de l'arrondissement historique un secteur autonome à visiter. Les premiers guides consacrés exclusivement au Vieux-Montréal apparurent en effet dans les années 1960. Les ballades historiques proposées dans les guides montraient généralement aux visiteurs un Vieux-Montréal qui puisait dans les évènements historiques montréalais. Dans les rues arpentées, les vestiges que le visiteur trouvait sur son chemin étaient peu nombreux, car disparus depuis longtemps, même s'ils dépassaient les 18 monuments historiques que l'inventaire de 1960 avait recensés. Les parcours s'organisaient autour de rues, de places et d'édifices que le promeneur était invité à découvrir. En 1964, l'Office municipal de tourisme publiait ainsi un premier guide, Promenade dans le Vieux Montréal (Leduc, 1964). Eric McLean (1964), l'un des pionniers de la renaissance du quartier, faisait paraître Le passé vivant de Montréal. Léon Trépanier (1968), journaliste à La Patrie, livrait Les rues du Vieux Montréal au fil du temps, tandis que John David King exprimait sa vision du quartier avec Old Montreal. Then Now. En ce sens, les ouvrages perpétuaient une tradition instaurée par Victor Morin, qui avait proposé un premier circuit de visite de la fondation de Montréal et qui s'était servi comme «matière» des plaques commémoratives installées 25 ans plus tôt (Gordon, 2001 ; Morin, 1942). Toutefois, ces ouvrages (dont trois des auteurs étaient d'ailleurs membres de la Commission Jacques-Viger) tentaient tout de même, à des degrés différents, de rattacher le visiteur à ce qu'il voyait, que ce soit par des références architecturales, par l'évocation de gestes qui ont permis la conservation de tel ou tel édifice ou par de l'information pratique qui démontrait la vitalité nouvelle du quartier. Comme dans le parcours de Victor 
Morin, c'était avant tout sur la ville coloniale et préindustrielle qu'on mettait l'accent.

Le Vieux-Montréal montré aux visiteurs correspondait aux canons du monument historique de l'époque où les valeurs d'âge et d'histoire étaient valorisées. Ce quartier au passé pluriséculaire offrait une assise à la métropole qui se construisait dans le nouveau centre-ville et qui nécessitait bien des démolitions. Ainsi, cette mince bande de terre, qui avait été amputée de la partie au nord de la rue NotreDame pour permettre la construction du palais de justice et du gratte-ciel de la Banque canadienne nationale, se présentait comme le point d'ancrage à partir duquel tout avait commencé. Dans le petit guide intitulé Le Vieux Montréal, publié en 1965, Jean Vandenplas écrivait :

Exposition universelle, métro, Place Ville-Marie, gratte-ciel... voilà le Montréal aujourd'hui. Mais au cœur de cette métropole moderne, emprisonné par le fleuve Saint-Laurent et par les vastes immeubles dressés vers le ciel, se trouve un paradis terrestre, baigné par la nostalgie des jours enfouis. C'est le berceau de la ville, les restes de ce passé proche et lointain où Montréal n'était qu'une bourgade du nom de Ville-Marie.

Au même moment, Jean Drapeau (1964), grand promoteur de la métropole moderne, soulignait : «Le passé survit dans les monuments et les anciennes constructions que nous tenons à préserver, dans le cachet et l'atmosphère de ce 'Vieux Montréal' où tout rappelle les origines héroïques de notre ville.» Les thèmes des origines et du commencement faisaient écho aux promesses de progrès et d'avenir des projets de renouveau urbain.

La décennie des années 1960 fut fondamentale dans la renaissance du Vieux-Montréal. La reconnaissance de l'importance du cadre bâti ancien, dont le bassin d'objets recensés s'élargit considérablement jusqu'à aujourd'hui, transforma de manière durable l'image de la ville. L'intégration des dimensions historique et culturelle, aux côtés du dynamisme économique et de la modernité métropolitaine, est aujourd'hui au cœur de l'identité urbaine. La mise en valeur du Vieux-Montréal fut déterminante dans ce processus. Petit à petit, grâce à une offre muséale bonifiée, à des circuits d'interprétation et à l'ouverture de commerces pour répondre aux besoins des nouveaux usagers (antiquaires, galeries d'art, librairies, boutiques, restaurants et bars), les visiteurs purent profiter d'une expérience urbaine innovante. Un véritable tourisme culturel se structurait. Le quartier devint ainsi la locomotive de l'industrie touristique dans la métropole. Enfin, sans l'apport et les promesses du tourisme, il n'est pas certain que le Vieux-Montréal serait ce qu'il est aujourd'hui. Il semble important de le rappeler.

\section{Bibliographie}

Archives de la Ville de Montréal (1965), VM34, Commission Jacques-

Viger, Procès-verbal, $40^{\mathrm{e}}$ réunion, 11 janvier.

Archives de la Ville de Montréal (1965), VM34, Commission Jacques-

Viger, Procès-verbal, $45^{\mathrm{e}}$ réunion, 17 mai.

AYOTTE, Alfred (1964), «Mains libres au Vieux Montréal», Le Presse,

11 janvier.
DRAPEAU, Jean (1964), «Préface», dans Clayton Gray, Montréal qui disparaît, Montréal, Éditions du Jour.

DROUIN, Martin (2008), «La mise en valeur du Vieux-Montréal : usage, histoire et architecture (1962-1979) ", dans Capucine Lemaître et Benjamin Sabatier (dir.), Patrimoine et patrimonialisation : fabrique, usage et réemploi, Québec, Éditions MultiMondes.

GORDON, Alan (2001), Making Public Pasts. The Contested Terrain of Montreal Public Memories, 1891-1930, Montréal / Kingston, McGill / Queen's University Press.

KING, John David (1969), Old Montreal. Then Now. Walking Tour, Montréal, Thérien Frères.

LALIBERTÉ, Jean-Marc (1961), «Le 'Vieux-Montréal' disparaîtra-t-il à tout jamais sous les gratte-ciel?», Le Devoir, 12 décembre.

LEDUC, Paul (1964), Promenade dans le Vieux-Montréal, Montréal, Office municipal de tourisme.

LUCHAIRE, André (1966), «La SHM dévoile un projet de 'Musée historique de Montréal'», Le Presse, 25 novembre.

MCLEAN, Eric et Robert D. WILSON (1964), Le passé vivant de Montréal / The Living Past of Montreal, Montréal, McGill University Press.

MONDELLO, Roméo (1961), Inventaire des maisons historiques dans le Vieux-Montréal, Montréal, Service d'urbanisme.

MORIN, Victor (1942), Le Vieux Montréal : fondation, développement, visite, Montréal, les Éditions des Dix.

PALMER, Al (1961), «Across Street - Back Two Centuries», The Gazette, 15 juillet.

PRÉVOST, Roland (1963), «Les musées et l'Expo 67 : Projets de musées dont Montréal a besoin pour son prestige et son avenir », La Presse, 21 février.

RAMACIERI, Anita (2008), Le Vieux-Montréal 2017, L'art de vivre montréalais dans une collection historique inscrite dans la modernité, Montréal, Bureau du patrimoine, de la toponymie et de l'expertise de la Ville de Montréal, [http://www.vieux.montreal.qc.ca/images/pdf/ vision2017.pdf], consulté le 6 janvier 2009.

RAMSAY, Lucie (2003), L’image de Montréal dans les guides touristiques, mémoire de maîtrise en études urbaines, Montréal, Institut national de la recherche scientifique - Urbanisation, culture et société.

Sans auteur (1963a), «Aménager Place Royale, berceau de la fondation de Montréal, un musée municipal ", Le Devoir, 26 juin.

Sans auteur (1963b), "Que sera le Vieux-Montréal en '68 ? », L'est central, 20 avril.

Société historique de Montréal (1917), Aux origines de Montréal, Mémoires de la Société historique de Montréal, onzième livraison, Montréal, Adj. Ménard.

TRÉPANIER, Léon (1968), Les rues du Vieux-Montréal au fil du temps, Montréal, Fides.

VANDENPLAS, Jean (1965), Le Vieux Montréal, Montréal, Fides. 